\title{
A MONTE CARLO PROGRAM FOR THE INVESTIGATION OF SCATTERING EFFECTS IN UNIFORM-FIELD MAGNETIC $\beta$-SPECTROMETERS *
}

\author{
M.S. HATAMIAN, T.A. GIRARD ** and M. SKALSEY \\ Randall Laboratory of Physics, University of Michigan, Ann Arbor, MI 48109, USA
}

Received 5 April 1983 and in revised form 2 September 1983

A Monte Carlo computer code which approximates the scattering and transmission of charged particles in a homogeneous sector field magnetic spectrometer is described. Program operation, including the function of required subroutines, is discussed. A comparison of calculated and experimental results yields reasonably good agreement, with single surface scattering events contributing to the transmission at the level of $1 \%$.

\section{Introduction}

In the recent development of a new technique for the precision relative measurement of the longitudinal polarization of positrons in nuclear $\beta$ decay [1], the difference in the transmission of spectrometer-scattered particles from comparison nuclei constitutes a potentially significant source of systematic uncertainty as a result of the differential depolarization of the particles upon backscattering [2]. The uncertainty becomes acute as the difference in endpoint energies between the comparison nuclei increases, and requires a careful examination in order to accurately assess its contribution to polarization measurements eventually anticipated to reach the level of better than $10^{-3}$ in accuracy. Such accuracy is required with the increasing demand for improved experiments testing electroweak theories at low momentum transfer, and is not confined to the measurement of $\beta$ polarization.

The study of such scattering effects is equivalent in principle to establishing a response function for the spectrometer system, and suggests the application of Monte Carlo methods as a means of providing guidance to the conduct of an experimental investigation. Frequent applications of these methods have previously included the modeling of $\gamma$-ray detector response functions [3] and beam transport calculations [4], each of which entails the tracking of particles through matter utilizing known scattering and absorption cross sec-

* Supported by DoE contract DoE-C-79ER10451, NSF Grants PHY-8109269 and PHY-8107573, and a grant from the Office of the Vice President for Research of the University of Michigan.

** Current address: Institute de Physique, Université de Louvain, Louvaine-la-Neuve, Belgium 1348. tions. In contrast, applications to the field focusing and transmission of charged particles appears to have received relatively little attention in the literature [5], and the application to effects of spectrometer scattering has not been considered.

In this report, a preliminary Monte Carlo computational program is described which simulates the transmission of positrons scattered from various surfaces of an asymmetric double-focusing flat-field sector spectrometer. This specialized configuration, in which one of the foci is located outside the spectrometer field, is required in order to deliver particles to the polarimeter. Although developed to assist in the identification of the response of this specific system at $182 \mathrm{G}(350 \mathrm{keV}$ positrons), the methods of calculation described are sufficiently general to be adapted to more standard configurations operated over a full range of field intensities. A comparison of computed and experimental results yields reasonably good agreement at precisions of better than $10^{-2}$, and suggests that the essential features of the various scattering processes have been incorporated, despite the use of rather naive approximations in the construction of the model.

\section{General description of calculation}

Particles are isotropically emitted from a distributed (planar) source and their trajectories individually computed in accordance with the equations of motion in a uniform magnetic field. Each trajectory is analyzed for a first intersection with either the detector or one of the spectrometer surfaces. For the $k$ th surface, an intersection probability relative to emission is determined from

$W_{k}^{\mathrm{inc}}(E, \boldsymbol{r})=\frac{N_{k}^{\mathrm{inc}}(E, \boldsymbol{r})}{N_{0} W_{\beta}(E)}$. 
where $N_{k}^{\text {inc }}(E, \boldsymbol{r})$ is the number of particles of energy $E$ incident on a spatial distribution $S(\boldsymbol{r})$ which describes the surface. $N_{0}$ is a fixed number of particles emitted from the source with energy $E$, which is weighted by the probability of emission $W_{B}(E)$ consistent with the spectral distribution of the nucleus under examination. $W_{k}^{\text {inc }}$ in effect describes the normalized particle illumination of the surface.

A large number of particles, $N_{k}^{\text {emit }}\left(E^{\prime}, r\right)$, is subsequently reemitted from each surface consistent with the calculated illumination distributions, as well as energy loss $(\Delta E)$ on scattering, $E^{\prime}=E-\Delta E$, and tabulated backscattering coefficients, $b_{k}(\theta)$. The new trajectories are computed, and analyzed for a next intersection. Reemitted particles incident on any non-detector surface are considered absorbed, and nonannihilating such that detector spectra are free from $\gamma$-contamination.

For each type of surface scattering, the probability of reaching the detector is determined by

$$
W_{k}^{\mathrm{det}}\left(E^{\prime}\right)=\frac{N_{k}^{\mathrm{det}}\left(E^{\prime}\right)}{\int_{S} N_{k}^{\mathrm{emit}}\left(E^{\prime}, \boldsymbol{r}\right) \mathrm{d} \boldsymbol{r}},
$$

where $N_{k}^{\text {det }}\left(E^{\prime}\right)$ is the number of particles of energy $E^{\prime}$ emitted from the $k$ th surface which reach the detector. The total number of particles scattered from the $k$ th surface which contribute to a transmission spectrum is then given by

$$
\begin{aligned}
N_{k}^{\text {det }}= & \int N_{k}^{\text {det }}\left(E^{\prime}\right) \mathrm{d} E^{\prime} \\
= & \iint W_{k}^{\text {det }}\left(E^{\prime}\right) b_{k}(\theta) W_{k}^{\text {inc }}\left(E^{\prime}+\Delta E, r\right) \\
& \times W_{\beta}\left(E^{\prime}+\Delta E\right) N_{0} \mathrm{~d} \boldsymbol{r} \mathrm{d} E^{\prime}
\end{aligned}
$$

\section{Description of the model}

\subsection{Spectrometer}

The spectrometer system, shown schematically in fig. 1 , consists of an air-cooled mass spectrometer of 12.70 $\mathrm{cm}$ radius with modified bending angle of $125^{\circ}$. The pole exit plane presents a $30^{\circ}$ angle to the exiting central ray, in order to provide horizontal and vertical foci for the beam at $0.8 \mathrm{~m}$ from spectrometer exit. The vacuum chamber is constructed from brass, and is fitted with five sets of $0.64 \mathrm{~cm}$ thick graphite slits arranged as shown. Each set alternately restricts either the vertical or radial beam trajectory, with the exception of the first set which restricts both. The chamber additionally contains an aluminum source holder and interchange mechanism, and graphite shields which prevent the transmission of particles from the source not under scrutiny.

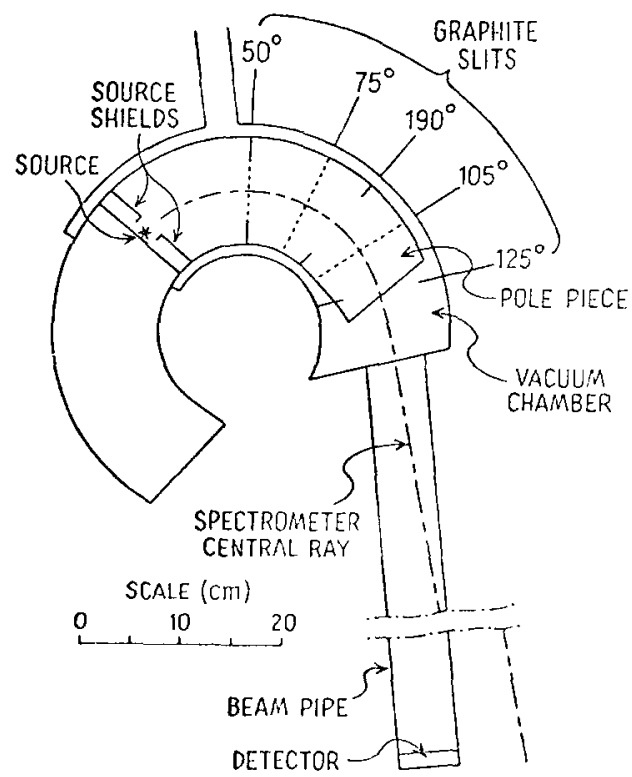

Fig. 1. Diagram of spectrometer system. A $125^{\circ}$ flat-field spectrometer with a $30^{\circ}$ exit plane has been modelled. A beam exits the spectrometer and is focused $\sim 1 \mathrm{~m}$ away. Graphite slits and shields are used to minimize backscattering effects.

\subsection{Approximations}

For the calculations presented, several simplifying approximations have been made. The spectrometer field is assumed homogeneous everywhere within the magnet gap, and zero everywhere without, such that the particle equations of motion are given by

$$
\frac{\mathrm{d}^{2} \boldsymbol{r}}{\mathrm{d} t^{2}}=\frac{e}{m} \boldsymbol{v} \times \boldsymbol{B},
$$

where $B$ is the magnetic field strength, and $e, m, r(t)$ and $v(t)$ represent the particle charge, mass, displacement, and velocity, respectively. The beam pipe and detector are defined by vertical planes only since neglect of fringing fields precludes any vertical focusing of the beam upon spectrometer exit. Particles exiting the spectrometer are only tracked horizontally thereafter, and intercepted by detector or beam pipe of infinite vertical extent. The detector, experimentally a silicon surface barrier detector, is modeled with a response function approximation consisting of a Gaussian peak about the incident energy plus constant fraction low energy tail [6].

Backscattering coefficients $(2 \pi)$ and angular distributions are obtained from MacKenzie et al. [7] and Seliger [8]. The energy of backscattered particles is determined from Baltakmen's empirical relationship between the maximum energy of a $\beta$ distribution before and after scattering [9],

$$
E^{\prime}=E(158+Z) / 251 \text {, }
$$


where $Z$ is the atomic number of the backscattering material.

A probability exists for particle transmission through the spectrometer slit baffling, since these are tapered at their edges. This was modeled using the angular distribution of complete diffusive scattering [10], of the form

$W(\theta) / W(0)=(0.717+\cos \theta) \cos \theta$,

where $W(\theta)$ is the probability of scattering into an angle $\theta$ within $\mathrm{d} \theta$. The energy loss is assumed to occur solely through ionization [11], for which

$$
\left(\frac{\mathrm{d} T}{\mathrm{~d} x}\right)=k \frac{E_{0}}{\beta^{2}}(N Z)\left[\ln \beta\left(\frac{T+E_{0}}{1}\right)\left(\frac{T}{E_{0}}\right)^{1 / 2}-\frac{1}{2} \beta^{2}\right] .
$$

The particle kinetic (rest) energy is given by $T\left(E_{0}\right)$, $\beta=v / c, k=1 \mathrm{~b} /$ electron, $(N Z)$ is the number of electrons per $\mathrm{cm}^{3}$ of the material, and $I=(11) Z \mathrm{eV}$ is the geometric mean of all ionization and excitation potentials of the absorbing atom.

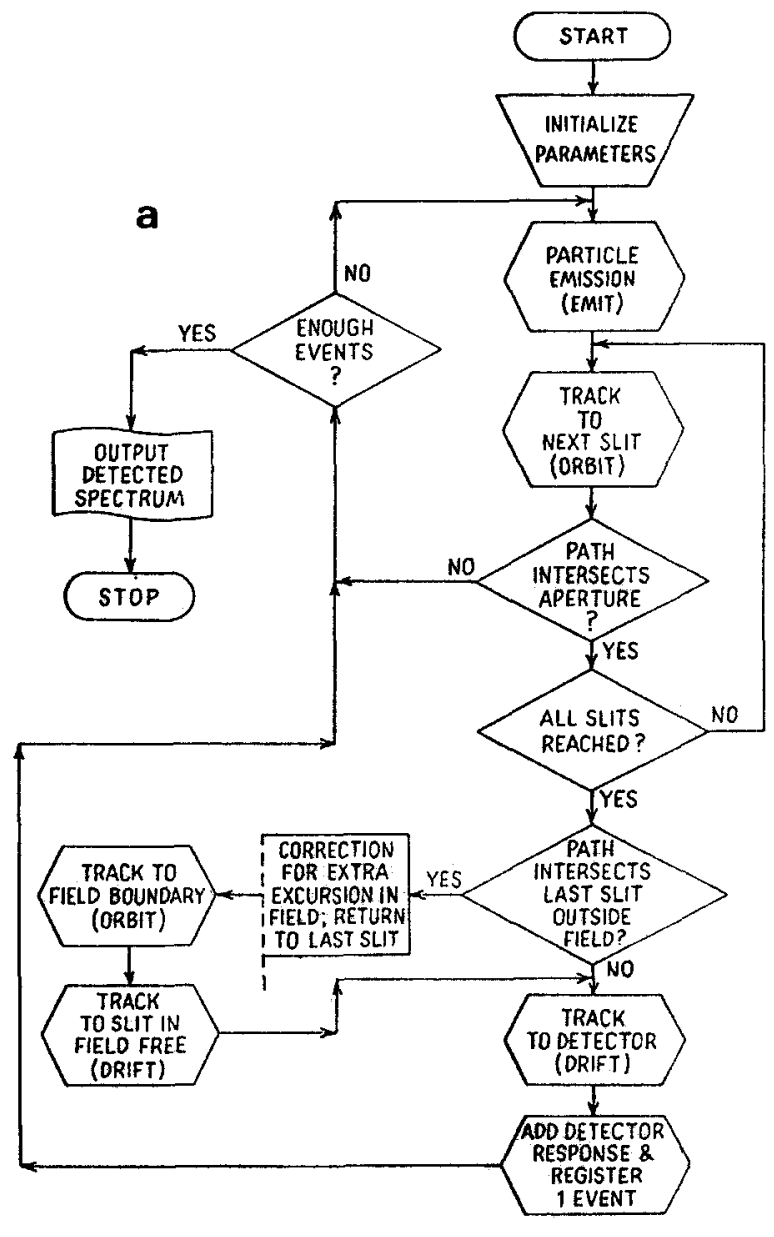

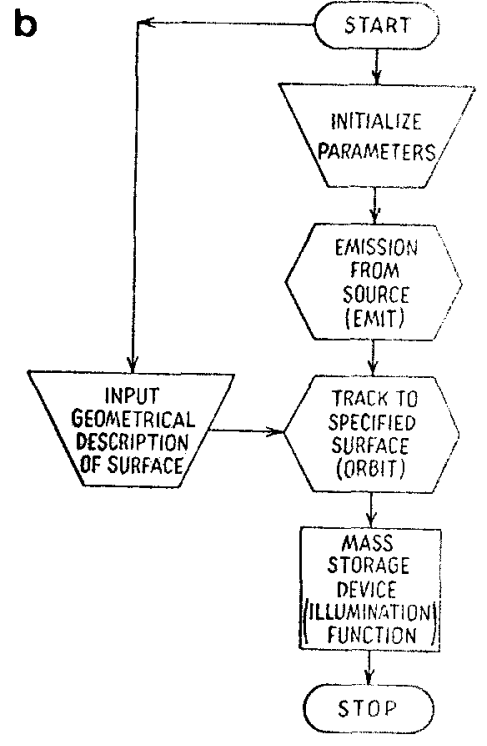

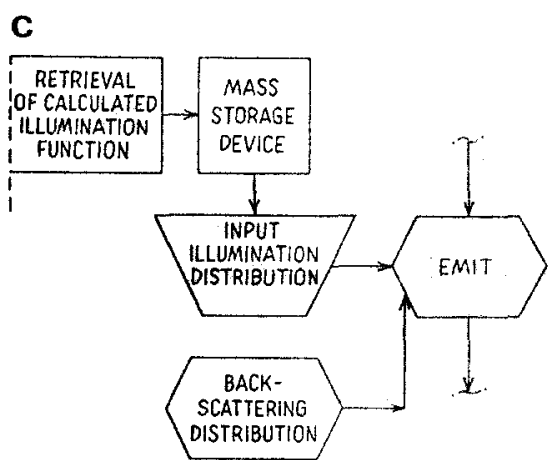

Fig. 2. Flow chart of program operation. (a) Transrnission: particles are emitted isotropically from a distributed source, and the trajectories tracked to determine if the detector is reached without scattering. (b) Illumination of spectrometer surfaces: emitted particles are tracked to determine the number, location and energy of those which strike a given surface. (c) Contribution of scattered particles to the transmitted beam: using the computed illumination distribution of each surface, scattered particles are tracked to determine if the detector is reached. This routine is identical to (a) above, except that the "EMIT" block is replaced by this flow chart.

\section{Program operation}

The flow chart of operation is presented in fig. 2. A Cartesian geometry is utilized, with the $x y$-plane defined perpendicular to the field direction. The program algorithm, described in section 2, is contained in MAIN, and is executed in two stages in order to obtain the total transmission $\left(N_{\mathrm{T}}\right)$ as

$$
\begin{aligned}
N_{\mathrm{T}} & \equiv \int N_{\mathrm{T}}(E) \mathrm{d} E \\
& =\int U(E) \mathrm{d} E+\sum_{k} \int N_{k}^{\mathrm{det}}\left(E^{\prime}\right) \mathrm{d} E^{\prime} .
\end{aligned}
$$


where $U(E)$ is the number of unscattered particles of energy $E$ reaching the detector.

\subsection{Illumination and transmission}

In this stage, $U(E)$ and the illumination distributions for each spectrometer surface are determined. The magnetic field strength, interest range of particle energies, source dimensions, desired number of particle emissions, and seed for the random number generator are accepted as input parameters. Each surface is examined separately.

An illumination file, consisting of an $n \times n$ array each element of which corresponds to a small area of the surface, is created in memory. Subroutine EMIT is called to randomly establish the initial coordinates of particle emission from a distributed source. For each particle, a random direction of emission is further generated from which the momentum components are computed consistent with the initial energy. For large numbers of events, the distribution of emissions is costrained to uniformity over the source surface, and isotropy within a forward hemisphere of $2 \pi$ sr. A $4 \pi$ emission distribution is achieved hy including the negative sign of the particle $x-y$ momenta. Both the location and momentum of emission are returned to MAIN.

A version of subroutine ORBIT specific to the surface under scrutiny is then called to determine if the particle intersects the surface. This is a generic routine which accepts as input particle coordinates and momenta, and computes the frequency and radius of the circular orbit. Various versions, all denoted as ORBIT in the flow chart, each contain a set of equations which describe the intersection of the particle trajectory with the geometrical definition of a specific surface. These are solved to define the $x-y$ coordinates of the interaction site. The time-of-flight to intersection is then determined, from which the intersection $z$-coordinate is computed. The routine returns the intersection coordinates of each particle to MAIN.

If intersection occurs with the surface under examination, the event is registered by incrementing the appropriate element in the illumination file. The program is then returned to EMIT and repeated until the desired number of events is reached, thus otaining $N_{k}^{\text {inc }}(E, r)$. A typical illumination distribution is shown in fig. 3. The particle emission energy is then incremented and the entire program repeated until the energy range of interest is spanned.

In the case of the first defining slit, subroutine EMIT is called to generate an emission, followed by ORBIT to determine if the particle intersects within the transmission aperture. If not, the slit illumination file is incremented and the program returned to EMIT. If the particle is within the transmission aperture, ORBIT is recalled and the process repeated for each succeeding

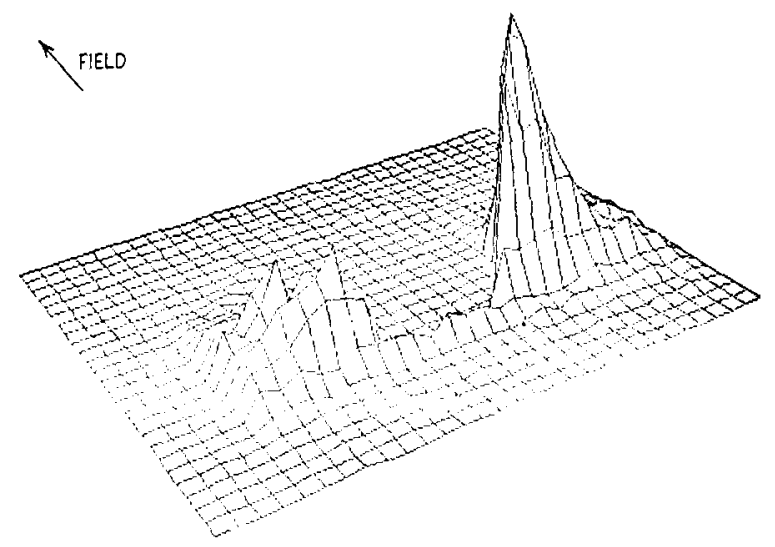

Fig. 3. Graphical display of a typical illumination file for the source interchange unit. Incident particle energy is $400 \mathrm{keV}$, with spectrometer field direction as shown. The observed distribution results from the circular hole in the aluminum plate, through which the source holder is introduced into the spectrometer. Similar files are created for each incident energy, and surface in the spectrometer.

slit until either the particle suffers an intersection or reaches the field boundary $(B=0)$.

If the boundary is reached, subroutine DRIFT is called to propagate the particle in the field-free region and determine whether its trajectory intersects the beam tube or detector. DRIFT accepts as input the location and momentum of the exiting particle, and intersects the trajectory determined from the equations of motion with the linear definition of the front surface of the detector. The energy and intersection coordinates of each particle are returned to MAIN, and the event is recorded.

The program returns to EMIT and the process repeated as described until both the desired number of total events is reached and the energy range of interest has been spanned.

\subsection{Scattering}

This stage computes the $N_{k}^{\text {det }}$, and operates similarly to the previous one with either subroutine REMIT or SLIT (see below) replacing EMIT. It also is executed separately for each surface under investigation. The number of events per surface element in each illumination file is multiplied by a common input factor to maintain the statistical accuracy obtained from the first stage.

For the initial energy and first surface element, REMIT is called to generate a scattering event. This routine, essentially a version of subroutine EMIT above, accepts as input a previously computed illumination distribution of a spectrometer surface under consideration, with the exception of the first slit. A large number of particle emissions is generated from the surface consistent with 
both the illumination distribution and backscattering coefficient. For each particle, a direction of emission is generated so that a large number of particles reproduces the angular distribution of backscattering. The energy of each backscattered particle is determined, and the momentum components are computed consistent with the energy and emission direction. Both the scattering energy and momentum are returned to MAIN, which then calls ORBIT to determine if the particle trajectory lies within the first slit transmission aperture.

Subroutine SLIT accepts as input the illumination distribution of the first slit edge. For each surface element, random emission directions into the edge are generated consistent with the angular distribution of complete diffusive scattering. The diffusion path is calculated in a number of steps, for which the momentum components of each step are computed consistent with incident energy and scattering direction. For each straight-line trajectory, the exit coordinates are determined and the energy loss by ionization in traversing the slit computed. The coordinates and momenta of particles exiting with nonzero kinetic energy are returned to MAIN.

In either case, the program continues to propagate the particle through the slit baffling using ORBIT as previously described until an intersection with either a spectrometer surface or detector is registered. The program then returns to REMIT (SLIT) and repeats the process until the weighted number of events has been reached. This is continued in sequence for each element in the surface matrix until complete. The energy is then incremented and the next illumination distribution processed until the desired range of particle energies is spanned.

\section{Comparison with experimental results}

All experimental data on the above spectrometer system were obtained using positrons from a $0.5 \mathrm{mCi}$ ${ }^{68} \mathrm{Ge} / \mathrm{Ga}$ source of dimensions $3 \mathrm{~mm} \times 2 \mathrm{~mm}$, and a 0.7 $\mathrm{mm}$ thick silicon surface barrier detector. The source thickness was approximately $4 \mathrm{mg} / \mathrm{cm}^{2}$. All calculations were performed on a VAX-11/780-VMS. The results required $6 \mathrm{~h}$ of CPU-time, and a total of $195 \mathrm{~kb}$ memory space of which $95 \mathrm{~kb}$ were allocated to illumination files.

Under the above approximations, the calculated transmission energy of an unscattered beam at $B=182$ $\mathrm{G}$ is $290 \mathrm{keV}$, with an energy resolution of $(2.0 \pm 0.1) \%$. In comparison, the experimental transmission energy is $350 \mathrm{keV}$ at $B=182 \mathrm{G}$, with a $(4.0 \pm 0.2) \%$ resolution. The computed ratio of the total number of $\beta$-particles detected to the total $4 \pi \beta$ emission of the source is $(6.6 \pm 0.5) \times 10^{-6}$, in contrast with the experimental value of $(7.9 \pm 0.8) \times 10^{-6}$. Both the predicted lower transmission energy and better resolution can be qualitatively attributed to the neglected fringing field. Since a fringing field is in effect a continuation of the main field at reduced intensity, its inclusion will focus particles of higher energy to the detector position. For $B=182 \mathrm{G}$, the spectrometer is then sampling from an energy region of the ${ }^{68} \mathrm{Ga} \beta$ distribution nearer the intensity maximum, which for the above energies amounts to roughly a $15 \%$ increase in the calculated transmission ratio. While the resolution simultaneously deteriorates with the increase in transmission energy, the horizontal defocusing of the fringing field should tend to reduce the effect. This hypothesis was tested through addition of a linear fringing field approximation [12], which raised the transmission energy to 350 $\mathrm{keV}$ accompanied by a deterioration in resolution to $7 \%$. An equivalent approximation, in which the pole edge is extended a small distance at full field [13], yielded identical results. In each case, however, only the vertical field component was taken into account, so that vertical focusing effects of the beam were neglected. Use of these approximations is at best only an indication of the fringing field effect, since data taken at the pole edge near the source describes a strongly exponential field. The operational configuration of the spectrometer did not permit access to the field region near the exit pole edge-beam pipe interface, which is modified by the presence of a small amount of transformer iron, and a more detailed investigation of these effects could not be pursued.

In order to facilitate a graphical comparison of the structural features of the particle distributions which

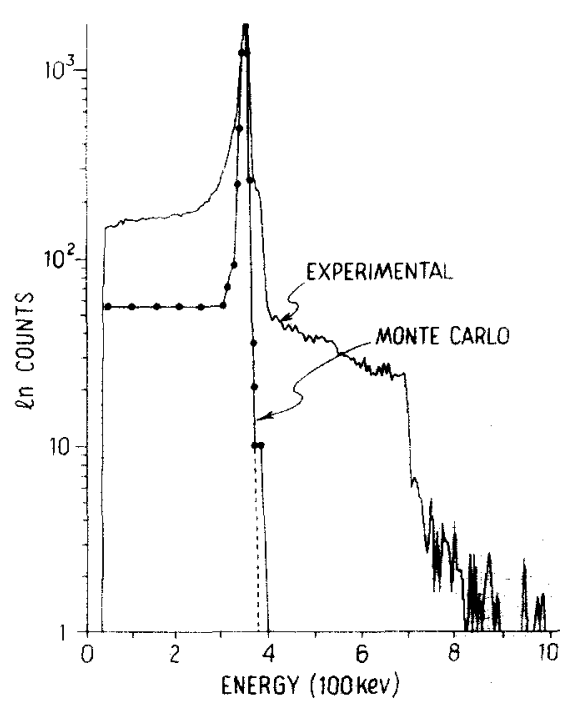

Fig. 4. Comparison of calculated and experimental spectrometer beams as viewed by a Si detector. The calculated spectrometer response function includes the unscattered, transmitted beam and all surface scattering contributions, as well as an approximate response function for the detector. 


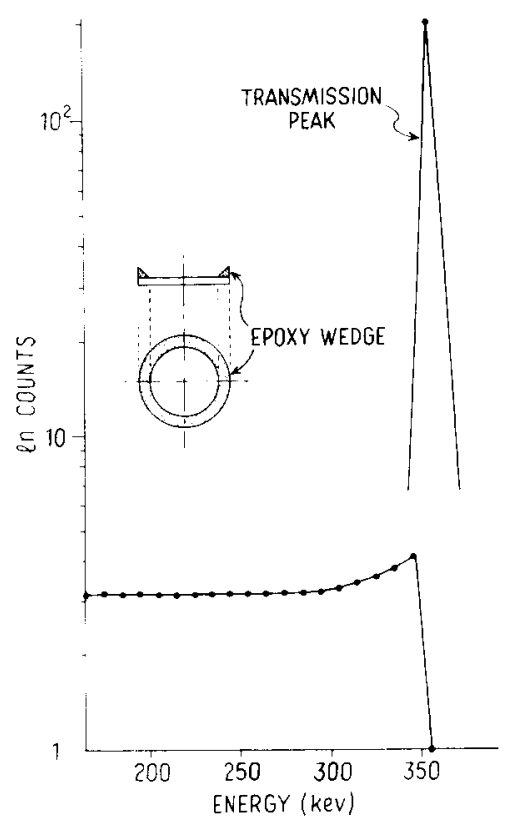

Fig. 5. Calculated effect of the epoxy on the Si detector front surface. The roughly constant low energy tail below the transmission peak is due to the energy loss by particles transiting the epoxy wedge (shown in insert) prior to reaching the detector.

partially accounts for the neglected fringing field effects, the computed distributions have been renormalized to the experimental transmission peak energy. This renormalization was accomplished by first matching the intensity of the computed transmission peak to the experimental, while preserving the structure-to-peak intensity ratios of the computed distributions in order to maintain the essential shape integrity. Each energy bin in the resulting distribution was then multiplied by a constant factor obtained from the ratio of experimentalto-calculated transmission peak energies. While this procedure results in a $20 \%$ increase in the $2 \mathrm{keV}$ bin width, all of the interesting structural effects occur within a relatively small energy interval and do not appear to suffer serious distortion. Nonetheless, all numerical comparisons are made using the unrenormalized calculations.

Fig. 4 is a summary comparison of calculated and experimental transmission spectra, with the computed spectrum containing all scattering contributions modeled to date, as well as the approximation to the detector response function. The agreement in the shape of these curves is quite good except in the region above $400 \mathrm{keV}$. This region experimentally contains events resulting from the summing of Compton-scattered annihilation radiation with the positron signal, and vanished under severe coincidence requirements which eliminated $\gamma$-contributions. While a reduction in the continuum below the transmission peak was also observed, difficulties in obtaining a Gaussian detector response during the coincidence experiments do not permit firm comments to be drawn regarding the predicted artifacts in the peak flanks which seem apparent experimentally.

While the theoretical detector response function contributes a roughly flat low energy tail to the spectra, the computed effect is insufficient in magnitude to reproduce experimental results. The detector front surface includes a thin wedge-shaped annulus of epoxy, shown in the insert to fig. 5. The effect of the glue was modeled

Table 1

Integrated illumination probabilities for each spectrometer surface

\begin{tabular}{lcc}
\hline Surface region & $\begin{array}{l}\text { Fraction of } 4 \pi \text { emission } \\
\text { illuminating surface } \\
\left(\times 10^{-2}\right)\end{array}$ & $\begin{array}{l}\text { Fraction of surface- } \\
\text { scattered events illuminating } \\
\text { detector }\left(\times 10^{-3}\right)\end{array}$ \\
\hline Source interchange unit & $12.00 \pm 0.01$ & $4.3 \pm 0.1$ \\
Behind shields & $9.50 \pm 0.01$ & $<0.01$ \\
Behind source & $28.50 \pm 0.01$ & $<0.01$ \\
Shield edge (large $r)$ & $3.0 \pm 0.5$ & $<0.1$ \\
Shield edge (small $r$ ) & $6.5 \pm 0.5$ & $6.1 \pm 0.1$ \\
Chamber walls: & $16.0 \pm 0.0$ & 0 \\
$\quad$ low energy spiralling & $15.4 \pm 0.4$ & $<0.1$ \\
$\quad$ source to slit 1 & $<0.1$ & $<0.1$ \\
beyond slit 1 a) & & $<0.1$ \\
Slit 1: & $6.60 \pm 0.01$ & $<0.1$ \\
graphite & $0.94 \pm 0.05$ & $10^{3}$ b) \\
tapered edge & $1.33 \pm 0.06$ & \\
transmission aperture & & \\
\hline
\end{tabular}

a) Includes slits 2-5.

b) This value is normalized using the absolute transmission magnitude of $6.6 \times 10^{-6}$ (Section 5). 


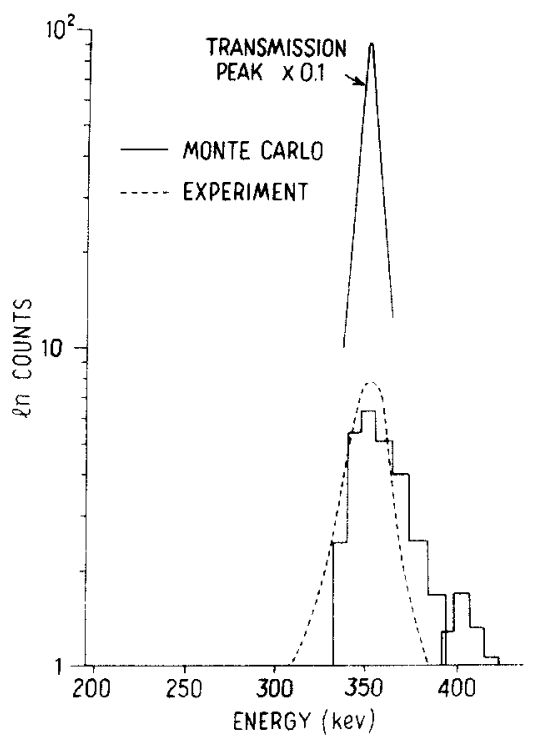

Fig. 6. Comparison of calculated and experimental spectra on the effect of backscattering from the source shields. Solid line histogram shows the calculated scattering contribution from the source shields. The inner shield yields the large effect directly under the transmission peak. The small, high energy satellite is due to scattering from the outer shield. Experimental conditions allowed measurements only on the inner shield, shown as the dotted line.

using diffusive-scattering distributions for particles normally incident on the detector. A low energy tail due to partial deposition is predicted as shown in fig. 5 , and is convolved with the backscattering effects in fig. 4. To observe this effect, the epoxy area of the detector was masked with a brass collimator. Spectra were obtained with detector masked and unmasked, and differences determined in the number of particles in a computersuggested energy region from $100 \mathrm{keV}$ to $25 \mathrm{keV}$ below the transmission peak. This region was found to contain $(20.5 \pm 1.0) \%$ of the transmission peak, within $2 \sigma$ agreement of the predicted value of $(18.3 \pm 1.4) \%$.

The energy- and geometry-integrated illumination probability of each spectrometer surface, and detection probability subsequent to scattering from each surface, is summarized in table 1 . Since the first and second column are, respectively,

$W_{k}^{\text {inc }}=\int W_{k}^{\text {inc }}(E, \boldsymbol{r}) \mathrm{d} E \mathrm{~d} \boldsymbol{r}$,

and

$W_{k}^{\text {det }}=\int W_{k}^{\text {det }}\left(E^{\prime}\right) \mathrm{d} E^{\prime}$,

a simple estimate of the number of detected particles scattered from the $k$ th surface is obtained from eq. (3) as

$N_{k}^{\text {det }} \approx W_{k}^{\text {inc }} b W_{k}^{\text {det }} N_{0} W_{\beta}$,

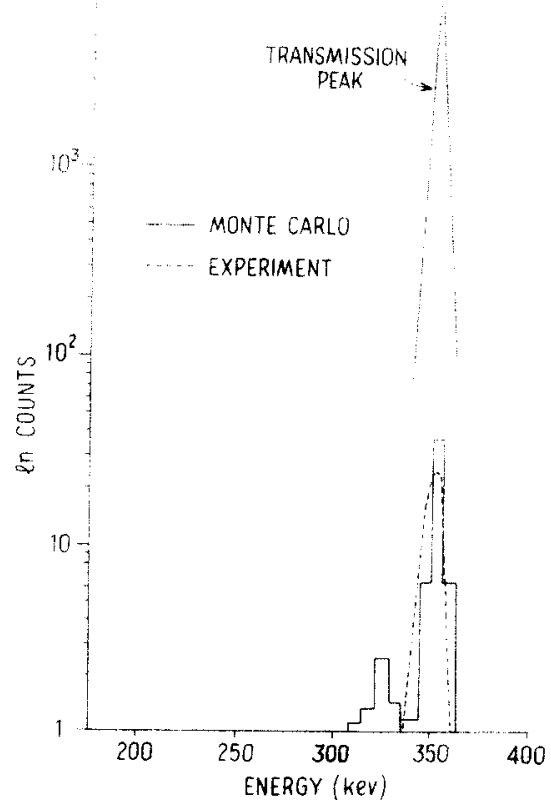

Fig. 7. Comparison of calculated and experimental spectra on the effect of backscattering from the source holder. Solid line histogram shows the calculated scattering contributions from the source holder. The outer part of the source holder yields the large effect directly under the transmission peak. The small, low energy satellite results from scattering on the inner part of the source holder. Experimental conditions allowed measurements on only the outer part, shown as the dotted line.

where $b$ denotes the backscattering coefficient integrated over scattering angle. Scattering from the chamber walls contributes essentially a flat distribution in the energy range of $300-420 \mathrm{keV}$, but at a significantly lower level than other effects. The entry labeled "low energy spiraling" describes particles emitted from the source with insufficient energy to reach the first slit transmision aperture following scattering from the chamber walls. Figs. 6 and 7 isolate the energy distributions of the predicted contributions from source interchange unit and shield scattering, respectively. In each case, the surfaces at large and small radial distances from the spectrometer center contribute differentially to the spectra, with the largest effects arising directly be-

Table 2

Fractional number of positrons scattered from various surfaces

\begin{tabular}{lcc}
\hline & \multicolumn{2}{c}{$N_{k}^{\text {det }}$} \\
& $\begin{array}{c}\text { Total transmission } \\
\text { Experiment }\end{array}$ & Calculation \\
\hline Source holder & $0.65 \pm 0.25$ & $0.9 \pm 0.1$ \\
Source shield & $0.5 \pm 0.7$ & $0.3 \pm 0.1$ \\
Lead plug & $13.0 \pm 0.3$ & $12.4 \pm 0.4$ \\
Graphite plug & $0.78 \pm 0.23$ & $1.1 \pm 0.3$ \\
\hline
\end{tabular}


neath the unscattered transmission peak. To further quantify these systematics, experimental investigations were conducted in which substantial areas of the aluminum source holder and graphite shields were separately masked with lead foil to enhance the backscattering. Transmission spectra with and without masking were obtained and differences in various computersuggested energy regions as shown in figs. 6 and 7 were measured. A summary of the results and comparison with prediction is contained in table 2 . All experimental uncertainties are statistical only. Also presented in table 2 are the results of two additional experiments in which a graphite plug, with and without lead masking of its front surface, was inserted into the source holder directly behind the source at a distance of $0.9 \mathrm{~cm}$.

\section{Discussion}

In the program results presented, no attempt has been made to quantitatively assess model-induced errors, and the uncertainties quoted are therefore statistical only. In table 2 , only the lead plug result does not suffer from poor statistical uncertainties when comparing experiment and calculation. The large rate enhancement $(\approx 12 \%)$ in backscattering in clearly seen in the experiment and agrees well with the expected value. The other effects, while in agreement, are much smaller $(\approx 1 \%)$ and do not severely test the program operation. The lead plug result, however, provides an indication that the program is operating as desired in spite of the numerous approximations employed in modeling the system and physical processes. The effect of scattering on the spectrometer response at $182 \mathrm{G}$ is to contribute to the transmission at the level of roughly $1 \%$ when low- $Z$ materials are used in the spectrometer construction.

The energy distribution of backscattered particles employed is clearly a gross oversimplification since it represents in effect only a shift of the decay spectrum to lower energy. Experimentally a backscattered $\beta$ distribution is both energy-degraded and shape-distorted, with a shift of particles towards lower energies. Use of the approximation is thus perhaps warranted only if the actual backscattering energy distribution of incident monoenergetic particles is significantly narrower than the $\beta$ decay spectrum under examination.

Although generally the case for high- $Z$ scatterers, the angular distribution broadens dramatically with decreasing $Z$ [14-16] and suggests that calculation of scattering effects arising from the aluminum and graphite surfaces may underestimate the contributions. Moreover, the backscattered angular distribution is differentially dependent upon incidence angle [14], which is not taken computationally into account since detailed experimental information regarding the angular back- scattering of $\beta$ 's as a function of $Z$ and incidence angle is not available. The majority of transmitted scattering events studied arise from particles incident within $35^{\circ}$ of normal, for which range a Monte Carlo study [17] indicates only a small variation of $b$. Furthermore, since the backscattered particles from any given spectrometer surface which contribute to the detected spectrum are incident within a small interval of angles $\left( \pm 5^{\circ}\right.$ about a central value) within this range, the contributions of such effects would appear to be negligible. A more detailed inclusion of this distribution should not be ignored, however, and further experimental investigations into both spectral and angular backscattering distributions would prove useful in this regard.

The most serious approximation involved, however, is the complete neglect of the magnetic fringing field, since it is precisely this field which provides the double-focusing character of the spectrometer. As noted earlier, our preliminary studies of such effects through various fringing field approximations indicate a general shift of the entire transmission spectrum towards higher energies with degradation of the resolution, as anticipated. While further quantitative examination of those effects was prohibited by the inaccessibility of the exit pole region to field probes, they do not appear to otherwise significantly modify the results of present calculations. Experiment does indicate a sharp vertical focusing of the beam at the detector location, such that inclusion of the proper beam pipe and detector vertical geometry is estimated to fractionally reduce the transmission by only several percent at most. Nevertheless, examination of such effects is essential to a complete understanding of the system behavior, and should not be neglected. In principle, they may be accounted for by a straightforward modification of the program to include a subroutine for the incremental calculation of the particle trajectories beyond the spectrometer exit plane using a detailed field map.

Finally, the present results do not include the effects of multiple surface scatterings of particles within the spectrometer. An approximate upper limit for such contributions is given by

$N_{i}<W_{i}^{\text {inc }} b_{i} W_{j}^{\text {inc }} b_{j} W_{j}^{\text {det }} N_{0} W_{\beta}$,

where the subscripts $i, j$ represent the $i, j$ th surfaces. As seen from the spectrometer geometry of fig. 1 together with consideration of the solid angles of the respective scattering surfaces, the inequality is valid for all surfaces with non-negligible $W^{\text {det }}$. From table 1, multiple surface scattering contributions are smaller than those of single surface scattering by one to two orders of magnitude, and when considered within the large uncertainties of the backscattering description employed are negligible in this phase of study. 


\section{Conclusions}

As evident from this work, the application of Monte Carlo techniques to $\beta$-spectrometers may prove a valuable tool for providing guidance to the experimental investigation of scattering systematics in $\beta$ spectrometers. As seen, reasonably useful information is obtained under a simple set of assumptions regarding the focusing and scattering of particles, and includes (1) an estimate of the magnitude of the scattering from the various spectrometer surfaces, and (2) the energy distribution of the particles that reach the polarimeter after scattering. The latter is of particular importance in the experimental measurement of the respective scattering contributions, since regions in the energy spectrum are indentified in which particles that have scattered from a given surface are likely to appear.

Modification of the program to other flat-field spectrometer configurations is easily accomplished, since only the geometrical specification of all scattered surfaces is required. Although in principle the methods of calculation can be applied to the study of any $\beta$-spectrometer system, both the application to nonuniform field-type spectrometers and inclusion of fringing fields will demand further effort. In order to reach the level of computational accuracy required to permit a significant contribution in $\beta$ spectroscopy, however, further quantitative information regarding the energy-, incidence angle-, and Z-dependencies of the backscattering distributions is needed.

We gratefully acknowledge useful discussions with D.E. Newman, M. Wiedenbeck, and D. Paul during this investigation. A significant contribution to the early stages of program development was made by $\mathrm{T}$. Bright.

\section{References}

[1] M.Skalsey, T.A. Girard, D.E. Newman and A. Rich, Phys. Rev. Lett. 49 (1982) 708.

[2] L. Braicovich, B. De Michelis and A. Fasana, Phys. Rev. 145 (1966) 952.

[3] I.M. Sobol, The Monte Carlo method (University of Chicago Press, Chicago, 1974);

F.T. Avignone, III, Nucl. Instr. and Meth. 174 (1980) 555 and references therein.

[4] L.L. Carter and E.D. Cashwell, Particle-transport simulation with the Monte Carlo method (ERDA Critical Review Series TID-26607, 1975).

[5] I.R. Williams, Nucl. Instr. and Meth. 60 (1968) 4; J.E. Spencer and H.A. Enge, Nucl. Instr. and Meth. 49 (1967) 181 ; I. Lindgren, B. Olsen, G. Petterson and W. Schneider, Nucl. Instr. and Meth. 41 (1966) 331

[6] M.J. Berger et al, Nucl. Instr. and Meth, 69 (1969) 181.

[7] I.K. Mackenzie, C.W. Shulte. I. Jackman and I.L. Campbell, Phys. Rev. A7 (1973) 135

[8] H.H. Seliger, Phys. Rev. 88 (1952) 408

[9] T. Baltakmens, Nucl. Instr. and Meth. 125 (1975) 169.

[10] G. Knop and W. Paul, in Alpha-, beta- and gamma-ray spectroscopy, ed., K. Siegbahn, vol. 1 (North-Holland, Amsterdam, 1965) p. 7.

[11] R. Evans, The atomic nucleus (McGraw-Hill, New York, 1955).

[12] H. Wollnik and H. Ewald, Nucl. Instr. and Meth. 36 (1965) 93.

[13] J.J. Livingood, The optics of dipole magnets (Academic, New York. 1969) p. 4.

[14] H. Frank, Z. Naturforschg. 14a (1959) 247

[15] P.L. Gruzin, Yu.V. Petrikin and A.M. Rodin, Atomnaya Energiya 33 (1972) 779.

[16] J. Jakshik and K.P. Jüngst, Nucl. Instr, and Meth. 79 (1970) 240.

[17] V.A. Kuziminikh and S.A. Vorobier. Nucl. Instr. and Meth. 129 (1975) 561. 